\title{
Universal adaptive control of satellite formation flying
}

\author{
R. PONGVTHITHUM†, S. M. VERESt, S. B. GABRIEL $\$$ and E. ROGERS§* \\ $\dagger$ Department of Mechanical Engineering, Chiang Mai University, Thailand \\ $\$$ School of Engineering Sciences and University of Southampton, Southampton SO17 1BJ, UK \\ $\S$ School of Electronics and Computer Science, University of Southampton, Southampton SO17 1BJ, UK
}

(Received August 2004; accepted November 2004)

\begin{abstract}
In this paper, a new universal adaptive control scheme for satellite formation flying is developed. The underlying idea of our design is to combine the domination design and the monotone adaptive gain. This scheme is guaranteed to have the properties of position tracking and full adaptivity against all parameters. Simulation studies are given which establish that implementation of this scheme would not require unachievable actuator signals.
\end{abstract}

\section{Introduction}

A new adaptive control law is developed for satellite formation flying which has recently become an important field of research in the space industry due to the benefits which can arise from this mode of operation. In particular, formation flying of several smaller satellites, instead of operating a single larger one, has the benefits of (i) a more cost effective synthetic aperture radar (SAR) for observations, (ii) graceful degradation: the failure of the on-board system on one satellite does not necessarily result in failure of the whole mission, (iii) increased flexibility since satellites can change/alter their specific roles, and (iv) smaller overall cost because of the reduced total mass put into orbit. SAR forms the basis of several missions to be launched by ESA (MicroSAR and TerraSAR programmes) and NASA (EO-1 programme). These advantages (Sabol et al. 2001) can only become available with the development and implementation of robust and reliable systems for controlling the formation. The on-board controllers should be the simplest possible and still must allow for the broadest class of manoeuvres with the maximum possible degree of efficiency.

The control of satellites in formation is currently the subject of much research effort in the control systems community at large (see, e.g. Leonard et al. 1999,

*Corresponding author. Email: s.m.veres@ecs.soton.ac.uk de Queiroz et al. 2000, Yeh et al. 2000), using a variety of configurations and control algorithms. For enhanced reliability, control topologies with decentralized capabilities are preferable. One possibility here is to replicate the same control hardware on each craft and allow the software to determine which satellite is the leader and which are followers. This paper addresses the generic problem of tracking a prescribed path by a follower relative to a leader satellite, and hence can be applied in various situations: for instance where there is a single leader and all the rest are followers or where each satellite follows the next in a chain.

For a cluster of satellites, the term 'formation keeping' is used to describe the control mechanism which is employed to keep them in fixed relative positions in either the inertial or the local coordinate system in orbit. The term 'manoeuvre' of one satellite relative to the other is used to describe the change in the relative position of two satellites in either of the coordinate systems. Clearly, what is formation keeping in one coordinate system could qualify for manoeuvre in another, and formation keeping in one coordinate system usually refers to the orbiting of a satellite around the leader in another coordinate system. By convention, the satellite that uses active control action is termed the slave or follower satellite and the other is termed the master or leader.

Figure 1 illustrates the coordinate systems involved and illustrates manoeuvres of satellites from position $\mathrm{B}, \mathrm{B}^{\prime}$ to positions $\mathrm{A}, \mathrm{A}^{\prime}$, respectively. The origin 
of each coordinate system is fixed to a leader satellite without active control, i.e. in natural orbit. Hence in formation keeping the controller of the follower also has to compensate for the disturbances acting on the leader.

Using the Hill (1878) equations, Sabol et al. (2001) investigated four classes (in-plane, in-track, and two circular ones) of stationary 'natural' formations in which pairs of satellites could, in principle, fly without control actuators. Simulations in Sabol et al. (2001), however, showed significant drift would always arise due to natural disturbances and concluded that active control action is needed to keep the satellites in any formation. In Veres et al. (2002) the robust constrained control problem was solved for circular orbits and McInnes (1993) has used artificial potential functions to guide the craft along a path and to ensure obstacle avoidance without parameter adaptation.

Using full non-linear dynamics instead of the Hill linearization, adaptive control has been developed by Wong et al. (2001). They used a nonlinear model included full non-linear dynamics, disturbances and leader control inputs. Including the leader, control meant that the model represented a much wider class of the leader's orbit, beyond Keplerian orbits. In Wong et al. (2001), asymptotic tracking has been proven in the case when the masses of the follower and leader satellites are unknown constants, and the disturbances are periodic with known periods, which is a restrictive assumption. Furthermore, the adaptive controller uses five-dimensional adaptive gain and depends

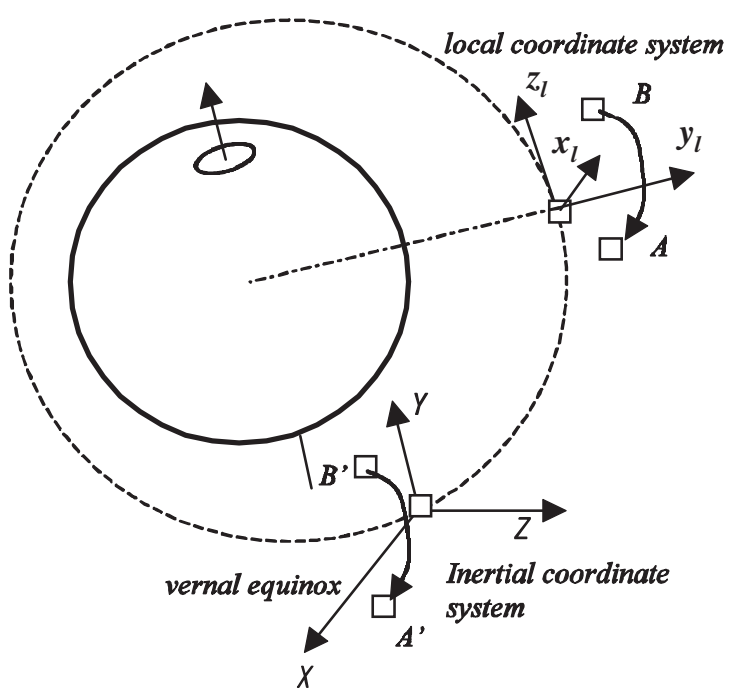

Figure 1. The generic control problems of actively controlling a follower satellite's position relative to the leader on a natural orbit, in either the inertial or local coordinate systems, depending on the purpose of the formation. Control laws for a cluster of satellites can be derived from a solution to this generic problem. on the leader position, orbit and control inputs, i.e. constant communication between the leader and the follower is needed.

Based on the non-linear model in Wong et al. (2001), the main objective in this paper is to develop an adaptive control scheme that further enhances the degree of adaptivity with respect to all parameters presented while minimizing the communication between both satellites and design complexity. The new features are as follows.

- The adaptive controller is independent of both satellites' parameters, the leader position, orbit and control inputs.

- All model parameters could be unknown and time varying. Allowing the model parameter to be time varying is important since for example, the masses of both satellites could be changed due to fuel consumption and the angular velocity $\omega$ and the angular acceleration $\dot{\omega}$ could be changed due to a non-circular orbit.

- Compared to the approaches based on Hill equations, the satellite cluster can move on an elliptic orbit as well as non-Keplerian orbits.

- No communication between both satellites is needed assuming that the follower can measure relative position and velocity. Hence, decentralized control can be implemented. This is particularly important when deploying a large fleet of satellites.

- The leader satellite can actually alter its orbit while the follower satellites track/maintain the formation. This is particularly important for Earth observations.

- The dimension of our adaptive gain is one which is the minimum.

- There is no requirement for the disturbances to be periodic.

The generic adaptive tracking solution developed here includes various formation flight topologies. In figure 2 the arrows indicate the tracking pairs of satellites. This paper focuses on the development of, and performance predictions for, a new algorithm for designing a universal adaptive formation flying controller and therefore obstacle avoidance and observer design are not discussed.

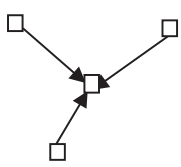

(a)

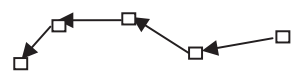

(b)
Figure 2. Tracking topologies: (a) star formation (b) chain formation. 


\section{Dynamical equations}

Consider the following set of equations describing the non-linear relative motion dynamics of two satellites (Wong et al. 2001)

$$
\begin{aligned}
\ddot{x}-2 \omega \dot{y}-\dot{\omega} y-\omega^{2} x+\frac{\mu x}{\|R+q\|^{3}}-\frac{u_{l} x}{m_{l}}-F_{d x} & =\frac{u_{x}}{m_{f}} \\
\ddot{y}+2 \omega \dot{x}+\dot{\omega} x-\omega^{2} y+\frac{\mu(y+r)}{\|R+q\|^{3}}-\frac{\mu r}{\|R\|^{3}}-\frac{u_{l y}}{m_{l}}-F_{d y} & =\frac{u_{y}}{m_{f}} \\
\ddot{z}+\frac{\mu z}{\|R+q\|^{3}}-\frac{u_{l z}}{m_{l}}-F_{d z} & =\frac{u_{z}}{m_{f}}
\end{aligned}
$$

where $R=[0, r, 0]^{\mathrm{T}}$ is the position vector from the inertial coordinate attached to the centre of the Earth to the leader satellite described in the local coordinate frame $\left\{x_{l}, y_{l}, z_{l}\right\}, q=[x, y, z]^{\mathrm{T}}$ is the position vector of the follower satellite relative to the leader satellite in the local coordinate $\left\{x_{l}, y_{l}, z_{l}\right\}, u_{l}=\left[u_{l x}, u_{l y}, u_{l z}\right]^{\mathrm{T}}$ is the control input of the leader satellite, $u=\left[u_{x}, u_{y}, u_{z}\right]^{\mathrm{T}}$ is the control input of the follower satellite, $\omega$ is the orbital angular velocity of the leader satellite, $\mu$ is the Earth's gravitational constant, $m_{l}$ and $m_{f}$ are the masses of the leader satellite and the follower satellite respectively, and $F_{d}(t)=\left[F_{d x}(t), F_{d y}(t), F_{d z}(t)\right]^{\mathrm{T}}$ is a bounded unknown time-varying disturbance.

Here we consider the problem of adaptive position tracking of the relative position of the follower satellite. Specifically, we concentrate on the following control problem:

Problem of adaptive practical position tracking: Given a constant $\varepsilon>0$ and a bounded reference signal $q_{r}(t)=\left[x_{r}(t), y_{r}(t), z_{r}(t)\right]^{\mathrm{T}} \in C^{1}$ whose derivative is also bounded, find, if possible a $C^{r}(r \geq 0)$ adaptive controller of the form

$$
\begin{aligned}
\dot{K} & =\eta\left(q, q_{r}(t)\right), \quad K \in \mathbb{R} \\
u & =\mu\left(q, K, q_{r}(t)\right), \quad u \in \mathbb{R}^{3}
\end{aligned}
$$

such that

(a) the states of the closed-loop system (1) and (2) are well-defined on $[0,+\infty)$ and bounded;

(b) there is a finite time $T_{\varepsilon}>0$ such that the closed-loop system (1) and (2) trajectories satisfy

$$
\left\|q(t)-q_{r}(t)\right\|<\varepsilon, \quad \forall t \geq T_{\varepsilon}>0 .
$$

Throughout this paper we make the following assumptions.
Assumption 1: There exists an unknown constant $M_{1} \geq 0$ such that

$$
\left\|q_{r}(t)\right\|+\left\|\dot{q}_{r}(t)\right\| \leq M_{1}, \quad \forall t \geq 0 .
$$

Assumption 2: There exists an unknown constant $M_{2} \geq 0$ such that

$$
|\omega(t)|+|\dot{\omega}(t)|+\left\|u_{l}(t)\right\|+\left\|F_{d}(t)\right\| \leq M_{2}, \quad \forall t \geq 0 .
$$

Assumption 3: There exists an unknown constant $m>0$ such that

$$
0<\left|m_{f}(t)\right|+\left|m_{l}(t)\right| \leq m, \quad \forall t \geq 0 .
$$

Note: In systems theoretic terms, it is also possible to treat $\mu$ as an unknown quantity.

It is worth mentioning that we only assume the boundedness property in Assumptions 2 and 3. We do not assume continuity. Hence, all of the parameters and functions discussed in Assumptions 2 and 3 could be discontinuous. This allows us to deal with a large class of parameters and functions and results in a more robust adaptive control law compared to Wong et al. (2001).

\section{Main result}

Theorem 1: Under Assumptions 1 and 3, the problem of adaptive practical position tracking of (1) is solved by the adaptive controller

$$
\left[\begin{array}{l}
u_{x} \\
u_{y} \\
u_{z}
\end{array}\right]=\left[\begin{array}{c}
\left(\begin{array}{c}
-\left[\bar{e}_{1}+(1+K) e_{1}\right]\left[1+K+K\left(\bar{e}_{2}^{2}+e_{2}^{2}+e_{1}^{2}\right)\right. \\
\left.+K^{3}\left(\bar{e}_{1}^{2}+1\right)+K e_{1}^{2}\right]
\end{array}\right) \\
\left(\begin{array}{c}
-\left[\bar{e}_{2}+(1+K) e_{2}\right]\left[1+K+K\left(\bar{e}_{1}^{2}+e_{1}^{2}+e_{2}^{2}\right)\right. \\
\left.+K^{3}\left(\bar{e}_{2}^{2}+1\right)+K e_{2}^{2}\right]
\end{array}\right) \\
-\left[\bar{e}_{3}+(1+K) e_{3}\right]\left[1+K+K^{3}\left(\bar{e}_{3}^{2}+1\right)+K e_{3}^{2}\right]
\end{array}\right]
$$

where $e=q-q_{r}, \bar{e}=\dot{q}$, the monotone non-decreasing function, $K(t) \geq 1$, is governed by

$$
\dot{K}= \begin{cases}\operatorname{sat}_{\beta}\left(\left\|q-q_{r}\right\|-\frac{\varepsilon}{2}\right), & \left\|q-q_{r}\right\| \geq \frac{\varepsilon}{2} \\ 0, & \left\|q-q_{r}\right\|<\frac{\varepsilon}{2}\end{cases}
$$

with $K(0)=1, \quad \forall \beta>0$

and the saturation function $\operatorname{sat}_{\beta}(s) \in \mathbb{R}$ is defined by 


$$
\operatorname{sat}_{\beta}(s)=\left\{\begin{array}{ll}
s, & |s| \leq \beta \\
\operatorname{sgn}(s) \beta, & |s|>\beta
\end{array} \quad \forall s \in R .\right.
$$

Proof: The proof is based on a modified version of the result in Lin and Pongvuthithum (2003) which combines the domination design and a monotone adaptive gain. In contrast to the result in Lin and Pongvuthithum (2003), which only deals with a high-order non-linear SISO system, here we show that this result can be extended to deal with the multi-input multi-output system (1).

First, we define a change of coordinates by

$$
e=\left[e_{1}, e_{2}, e_{3}\right]^{\mathrm{T}}=q-q_{r}, \quad \bar{e}=\left[\bar{e}_{1}, \bar{e}_{2}, \bar{e}_{3}\right]^{\mathrm{T}}=\dot{q} .
$$

Then using equation (10), the system (1) can be written as

$$
\begin{aligned}
\dot{e} & =\bar{e}-\dot{q}_{r} \\
\dot{\bar{e}} & =f(e, \bar{e})+g(q, r)+D(t)+\frac{1}{m_{f}} u
\end{aligned}
$$

where

$$
\begin{aligned}
& f=\left[\begin{array}{l}
f_{1} \\
f_{2} \\
f_{3}
\end{array}\right]=\left[\begin{array}{c}
2 \omega \bar{e}_{2}+\dot{\omega} e_{2}+\omega^{2} e_{1} \\
-2 \omega \bar{e}_{1}-\dot{\omega} e_{1}+\omega^{2} e_{2} \\
0
\end{array}\right], \\
& g=\left[\begin{array}{l}
g_{1} \\
g_{2} \\
g_{3}
\end{array}\right]=\left[\begin{array}{c}
-\mu x /\|R+q\|^{3} \\
-\left(\mu(y+r) /\|R+q\|^{3}+\mu r /\|R\|^{3}\right) \\
-\left(\mu z /\|R+q\|^{3}\right)
\end{array}\right] \\
& D=\left[\begin{array}{l}
D_{1} \\
D_{2} \\
D_{3}
\end{array}\right]=\left[\begin{array}{c}
\dot{\omega} y_{r}+\omega^{2} x_{r}+\left(u_{l x} / m_{l}\right)+F_{d x} \\
-\dot{\omega} x_{r}+\omega^{2} y_{r}+\left(u_{l y} / m_{l}\right)+F_{d y} \\
\left(u_{l z} / m_{l}\right)+F_{d z}
\end{array}\right] .
\end{aligned}
$$

Consider a Lyapunov function

$$
V(e, \xi)=\frac{1}{2} e^{\mathrm{T}} e+\frac{m}{2} \sum_{i=1}^{3} \xi_{i}^{2}, \quad \xi_{i}=\bar{e}_{i}+(1+K) e_{i} .
$$

Then the derivative of equation (14) along equation (11) satisfies

$$
\begin{aligned}
\dot{V}= & \sum_{i=1}^{3}\left[e_{i} \dot{e}_{i}+m \xi_{i}\left(f_{i}(e, \bar{e})+g_{i}(q, r)+D_{i}(t)\right)\right. \\
& \left.\left.+(1+K) \dot{e}_{i}+\dot{K} e_{i}\right)\right]+\frac{m}{m_{f}}\left(\xi_{1} u_{x}+\xi_{2} u_{y}+\xi_{3} u_{z}\right) .
\end{aligned}
$$

Using Assumption 1 and the completion of the squares technique, it is easy to show that

$$
\begin{aligned}
e_{1} \dot{e}_{1}=e_{1} \bar{e}_{1}-e_{1} \dot{x}_{r} & \leq e_{1} \bar{e}_{1}+\left|e_{1}\right| M_{1} \\
& \leq e_{1} \bar{e}_{1}+K e_{1}^{2}+\frac{M_{1}^{2}}{4 K} \\
& =-e_{1}^{2}+e_{1} \xi_{1}+\frac{M_{1}^{2}}{4 K} \\
& \leq \frac{-e_{1}^{2}+\xi_{1}^{2}}{2}+\frac{M_{1}^{2}}{4 K} .
\end{aligned}
$$

Applying the same process to the terms $i=2,3$, we have

$$
\sum_{i=1}^{3} e_{i} \dot{e}_{i} \leq \frac{1}{2} \sum_{i=1}^{3}\left(-e_{i}^{2}+\xi_{i}^{2}\right)+\frac{3 M_{1}^{2}}{4 K}
$$

and again using the completion of the squares technique, it follows that

$$
\begin{aligned}
m\left|\xi_{1} f_{1}(\cdot)\right| & \leq m\left|\xi_{1}\right|\left(2|\omega|\left|\bar{e}_{2}\right|+|\dot{\omega}|\left|e_{2}\right|+\omega^{2}\left|e_{1}\right|\right) \\
& \leq m\left|\xi_{1}\right|\left(2 M_{2}\left|\bar{e}_{2}\right|+M_{2}\left|e_{2}\right|+M_{2}^{2}\left|e_{1}\right|\right) \\
& \leq K \xi_{1}^{2}\left(\bar{e}_{2}^{2}+e_{2}^{2}+e_{1}^{2}\right)+\frac{m^{2}\left(4 M_{2}^{2}+M_{2}^{2}+M_{2}^{4}\right)}{4 K} \\
& =: \xi_{1}^{2} \hat{\rho}_{1}(e, \bar{e}, K)+\frac{\hat{\theta}_{1}}{K} \\
m\left|\xi_{2} f_{2}(\cdot)\right| & \leq m\left|\xi_{2}\right|\left(2|\omega|\left|\bar{e}_{1}\right|+|\dot{\omega}|\left|e_{1}\right|+\omega^{2}\left|e_{2}\right|\right) \\
& \leq m\left|\xi_{2}\right|\left(2 M_{2}\left|\bar{e}_{1}\right|+M_{2}\left|e_{1}\right|+M_{2}^{2}\left|e_{2}\right|\right) \\
& \leq K \xi_{2}^{2}\left(\bar{e}_{1}^{2}+e_{1}^{2}+e_{2}^{2}\right)+\frac{m^{2}\left(4 M_{2}^{2}+M_{2}^{2}+M_{2}^{4}\right)}{4 K} \\
& =: \xi_{2}^{2} \hat{\rho}_{2}(e, \bar{e}, K)+\frac{\hat{\theta}_{2}}{K} .
\end{aligned}
$$

Next, we derive an upper bound on the terms involving $K$ in equation (15), where using the fact that $K(t) \geq 1$, we obtain

$$
\begin{aligned}
& m \xi_{i}\left((1+K) \dot{e}_{i}+\dot{K} e_{i}\right) \\
& \quad \leq m\left|\xi_{i}\right|\left((1+K)\left(\left|\bar{e}_{i}\right|+M_{1}\right)+\beta\left|e_{i}\right|\right) \\
& \quad \leq m\left|\xi_{i}\right|\left(2 K\left(\left|\bar{e}_{i}\right|+M_{1}\right)+\beta\left|e_{i}\right|\right) \\
& \quad \leq \xi_{i}^{2}\left(K^{3}\left(\bar{e}_{i}^{2}+1\right)+K e_{i}^{2}\right)+\frac{m^{2}\left(4+4 M_{1}^{2}+\beta^{2}\right)}{4 K} \\
& \quad=: \xi_{i}^{2} \bar{\rho}_{i}(e, \bar{e}, K)+\frac{\bar{\theta}_{i}}{K}, \quad i=1,2,3 .
\end{aligned}
$$


To obtain an upper bound on the remaining terms in equation (15), we assume that for $i=1,2,3$, there exists an unknown constant $c$ such that

$$
\begin{aligned}
\left|g_{i}(q, r)\right| & \leq c \\
\left|D_{i}(t)\right| & \leq c .
\end{aligned}
$$

Then from Assumptions 1-3, equation (20) follows immediately. In general, equation (10) is not true, but in this particular application area it always holds since the radius of the leader satellite, $R$, is very large and, in particular, several orders larger than the relative position of the follower satellite, $q$. To give a rough idea, if the leader satellite is in the orbit at the attitude of $600 \mathrm{~km}$, it is easy to show that $\left|g_{i}\right| \leq 2, \forall\|q\| \leq$ $600 \mathrm{~km}$. With such a large margin of $q$ and relatively small bound, $q(t)$ can be easily guaranteed to stay within $600 \mathrm{~km}$ under a typical setup where $q_{r}(t)$ and $q(0)$ are only several kilometers. For a more concrete proof, Remark 2 provides a way to estimate the compact and invariant set containing $q(t), t \geq 0$. Thus, equation (19) can be verified.

Using equations (19) and (20), we now have that

$$
m\left|\xi_{i}\right|\left|g_{i}(q, r)+D_{i}(t)\right| \leq K \xi_{i}^{2}+\frac{\tilde{\theta}_{i}}{K}, \quad \tilde{\theta}_{i}=m^{2} c^{2}
$$

and combining equations (16), (17), (18), (21) and equation (15), yields

$$
\begin{aligned}
\dot{V}= & \sum_{i=1}^{3}\left[-\frac{1}{2} e_{i}^{2}+\xi_{i}^{2}\left(\frac{1}{2}+K+\rho_{i}(e, \bar{e}, K)\right)\right] \\
& +\frac{\theta}{K}+\frac{m}{m_{f}}\left(\xi_{1} u_{x}+\xi_{2} u_{y}+\xi_{3} u_{z}\right)
\end{aligned}
$$

where $\rho_{i}(\cdot)=\hat{\rho}_{i}(\cdot)+\bar{\rho}_{i}(\cdot) \geq 0 \quad$ with $\quad \hat{\rho}_{3}(\cdot)=0 \quad$ and $\theta=\left(3 M_{1}^{2} / 4\right)+\hat{\theta}_{1}+\hat{\theta}_{2}+\sum_{i=1}^{3}\left(\bar{\theta}_{i}+\tilde{\theta}_{i}\right)$ is an unknown constant independent of $K$.

Clearly the following choice of controllers

$$
\left[\begin{array}{l}
u_{x} \\
u_{y} \\
u_{z}
\end{array}\right]=\left[\begin{array}{l}
-\xi_{1}\left(1+K+\rho_{1}(\cdot)\right) \\
-\xi_{2}\left(1+K+\rho_{2}(\cdot)\right) \\
-\xi_{3}\left(1+K+\rho_{3}(\cdot)\right)
\end{array}\right]
$$

together with the fact that $\xi_{1} u_{x}, \xi_{2} u_{y}$ and $\xi_{3} u_{z} \leq 0$, yield

$$
\begin{aligned}
\dot{V} & =-\frac{1}{2} \sum_{i=1}^{3}\left(e_{i}^{2}+\xi_{i}^{2}\right)+\frac{\theta}{K} \\
& \leq-b V+\frac{\theta}{K}
\end{aligned}
$$

where $b=\min \{1,1 / m\}>0$.
In the remaining part of the proof, we show that all states of the closed-loop system (1)-(22)-(8) are bounded and well-defined on $[0,+\infty)$. Moreover, given any $\varepsilon>0$, there exists a finite time $T_{\varepsilon}$ such that the position tracking error $\|e\|=\left\|q-q_{r}(t)\right\| \leq \varepsilon$, $\forall t \geq T_{\varepsilon}$.

Using equation (23), we obtain

$$
\dot{V} \leq-b V+\theta
$$

which implies that $(e, \xi, K)$ are well-defined on $[0,+\infty)$ and $(e, \xi)$ is bounded. The compact set $\Omega=\{e, \xi \mid$ $V(e, \xi) \leq a, \forall a \geq \theta / b\}$ is invariant, since $V$ is positive definite and proper and $\dot{V} \leq 0, \forall e, \xi \in \Omega$. To show that $K(t)$ is bounded, we use a contradiction argument. In particular, suppose that the monotone nondecreasing function $K(t)$ is unbounded. Then there must exist a finite time $T^{*}$ such that

$$
K(t) \geq \frac{\theta}{\varepsilon^{*}}, \quad \varepsilon^{*}=\frac{b \varepsilon^{2}}{16}, \quad \forall t \geq T^{*}
$$

and equation (23) becomes

$$
\dot{V} \leq-b V+\varepsilon^{*}, \quad \forall t \geq T^{*} .
$$

Consequently,

$$
V(t) \leq e^{-b\left(t-T^{*}\right)}\left(V\left(T^{*}\right)-\frac{\varepsilon^{*}}{b}\right)+\frac{\varepsilon^{*}}{b}, \quad \forall t \geq T^{*} .
$$

This implies that there exists another finite time $T_{1}$ such that

$\frac{\left\|q-q_{r}(t)\right\|^{2}}{2}=\frac{e_{1}^{2}+e_{2}^{2}+e_{3}^{2}}{2} \leq V(t)<\frac{2 \varepsilon^{*}}{b}=\frac{\varepsilon^{2}}{8}, \quad \forall t \geq T_{1}$

which contradicts the assumption that $K(t)$ is unbounded since

$$
\dot{K}(t)=0, \quad \forall t \geq T_{1} .
$$

Since $K(t)$ is bounded, we can conclude that all of the states $(e, \bar{e}, K)$ of equations (11)-(22)-(8) are also bounded and well-defined. Hence, from Assumption 1, the closed-loop system trajectories generated by equations (1)-(22)-(8) are well-defined and bounded on $[0,+\infty)$.

To complete the proof, we show that the position tracking error $\|e\|=\left\|q-q_{r}(t)\right\|$ is eventually within the prescribed error $\varepsilon$ after a finite time. In particular, from equation (8), $\dot{K}(t)$ is uniformly continuous w.r.t. $e$ and by boundedness of the closed-loop states, $e$ is uniformly continuous w.r.t. time $t$. Hence, $\dot{K}(t)$ is uniformly 
continuous w.r.t. $t$. In addition, the integral of $\dot{K}(t)$ evaluated from zero to infinity exists and is finite, i.e.

$$
\lim _{t \rightarrow \infty} \int_{0}^{t} \dot{K}(\tau) \mathrm{d} \tau=K(\infty)-K(0)<+\infty
$$

Then, it follows from Barbalat's lemma that

$$
\lim _{t \rightarrow \infty} \dot{K}(t)=0
$$

This, together with equation (8), implies the existence of a finite time $T_{\varepsilon}$ satisfying

$$
\left\|q-q_{r}(t)\right\| \leq \varepsilon \quad t \geq T_{\varepsilon}>0 .
$$

Remark 1: If we assume that the follower satellite can measure its relative position $q$ and velocity $\dot{q}$ and the reference signal vector $q_{r}$ is given, the follower satellite is completely autonomous. No communication between the leader and the follower satellites is needed and the follower satellite can achieve relative position tracking regardless of the leader satellite's manoeuvres or orbits.

Remark 2: With the help of equation (24), the bound of $q(t)$ can be calculated since $e(t)$ only evolves in the compact set $\{(e, \xi): V(e, \xi) \leq \max \{V(e(0), \xi(0)), \theta / b\}\}$. By the definition of $e(t)$ and Assumption 1, we have $\|q(t)\| \leq \sqrt{2 \max \{V(e(0), \xi(0)), \theta / b\}}+M_{1}, \forall t \geq 0$. The bound of $q(t)$ can be used to verify (19) and guarantee that the closed-loop system (1)-(22)-(8) is well-posed, i.e. $R \neq-q$. It can be seen that in the satellite application, equation (19) is automatically satisfied and $R(t) \gg q(t)$ since $R(t)$ is several orders larger than the bounding constants, $M_{1}, M_{2}$ and $m$, and the initial conditions, $q(0)$ and $\dot{q}(0)$.

Note that the characteristics and performance of the adaptive controller (22) highly depend on the choice of the reference trajectory $q_{r}$. Choosing a wrong $q_{r}$ can cause unachievable control inputs or poor performance. However, path planning is not a trivial task and is beyond the scope of this paper. Instead, we show in the next section that for a simple manoeuvre, it is possible to choose simple trajectories to keep the size of control inputs within a range of small electric propulsion devices such as ion thruster, etc.

\section{Simulations}

In this section, we present a numerical simulation of a manoeuvre which requires moving the follower satellite from one relative position to another. We assume that the follower satellite can measure its relative position and velocity. The reference signal $q_{r}$ is described by

$$
q_{r}(t)=a \int_{0}^{t} \mathrm{e}^{-a(t-\tau)} Q_{r}(\tau) \mathrm{d} \tau
$$

where

$$
Q_{r}(t)= \begin{cases}\frac{X_{1}}{2}+\frac{X_{1}}{2} \sin \left(\pi\left(\frac{t}{T_{s}}-\frac{1}{2}\right)\right), & 0 \leq t \leq T_{s} \\ X_{1}, & t \geq T_{s} .\end{cases}
$$

Equation (26) can be viewed as a lowpass-filtered version of (27) which is a $C^{1}$ approximation of a square function. The purpose of equation (26) is to generate a signal to move the follower satellite from the origin to $X_{1}$ position and stay there afterwards while keeping the control input small and relatively smooth. The settling time $T_{s}$ determines how fast the follower satellite moves to the designated positions $X_{1}$.

The simulations was performed with the following parameters, $\quad m_{l}=m_{f}=4 \mathrm{~kg}, \quad a=0.01, \quad T_{s}=3600 \mathrm{~s}$, $F_{d}=[1.9106,-1.906,-1.517] \sin (2 \pi \omega t) \times 10^{-5} \mathrm{~m} / \mathrm{s}^{2}$, $X_{1}=[-100,100,100]^{\mathrm{T}}, q(0)=\dot{q}(0)=[0,0,0]^{\mathrm{T}}, \varepsilon=0.01$ and $\beta=10$. Initially, the leader satellite was an elliptic orbit with a perigee altitude of $600 \mathrm{~km}$ and an eccentricity of 0.2 . Then at $t=4800 \mathrm{~s}$, the leader satellite made a manoeuvre in the $y$-direction with the control input profiles shown in figure 5 .

The simulation results in figures 3-6 show that position tracking was achieved and the follower's control inputs were kept relatively small within the range of small electric thrusters during the whole
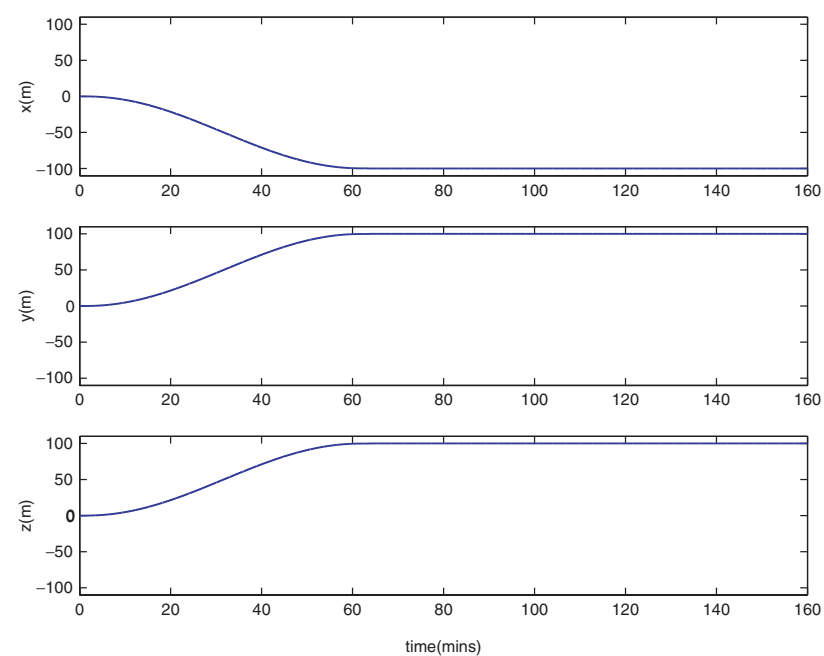

Figure 3. Relative position of the follower satellite. 

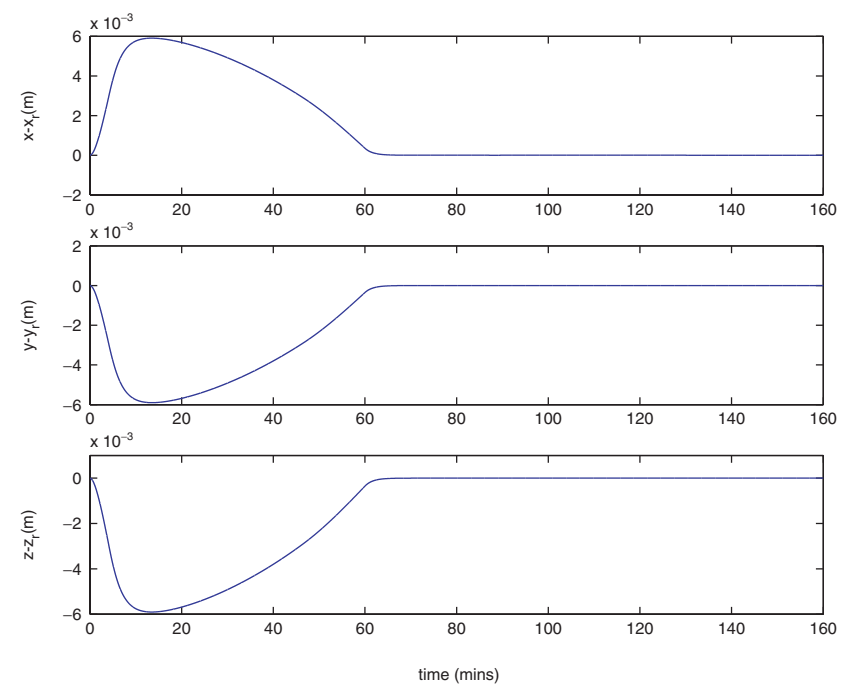

Figure 4. Tracking error of the follower satellite.
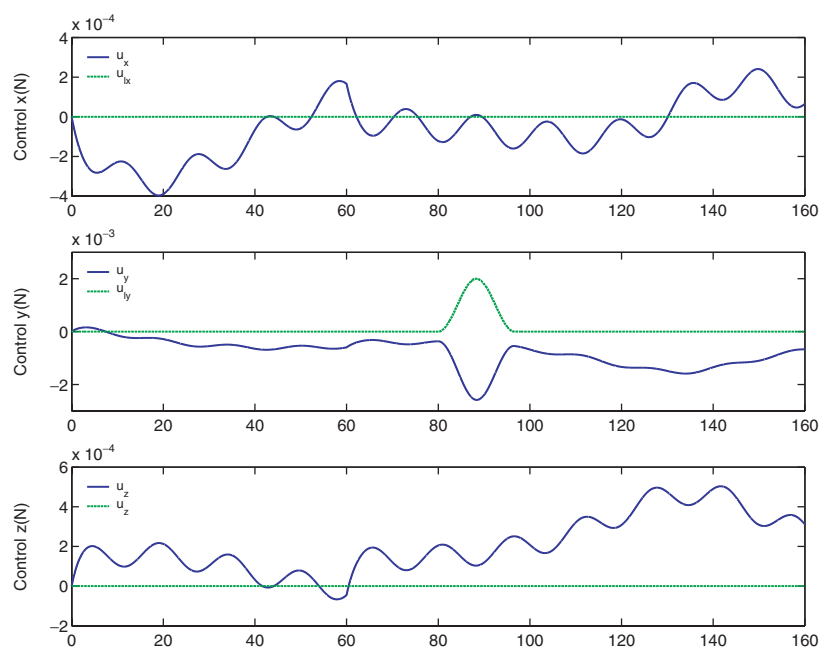

Figure 5. Control input of the follower (solid) and leader satellites (dot).

period. In addition, the relative error $e$ were maintained whilst the leader was making the manoeuvre without any predetermined information and communication.

\section{Conclusions}

Position tracking and full adaptivity against all parameters have been proven for the universal adaptive control scheme presented in this paper. Also the realistic small size of control thrust required is illustrated in the simulations. This new scheme is important for formation flying on elliptic orbits and also in cases where

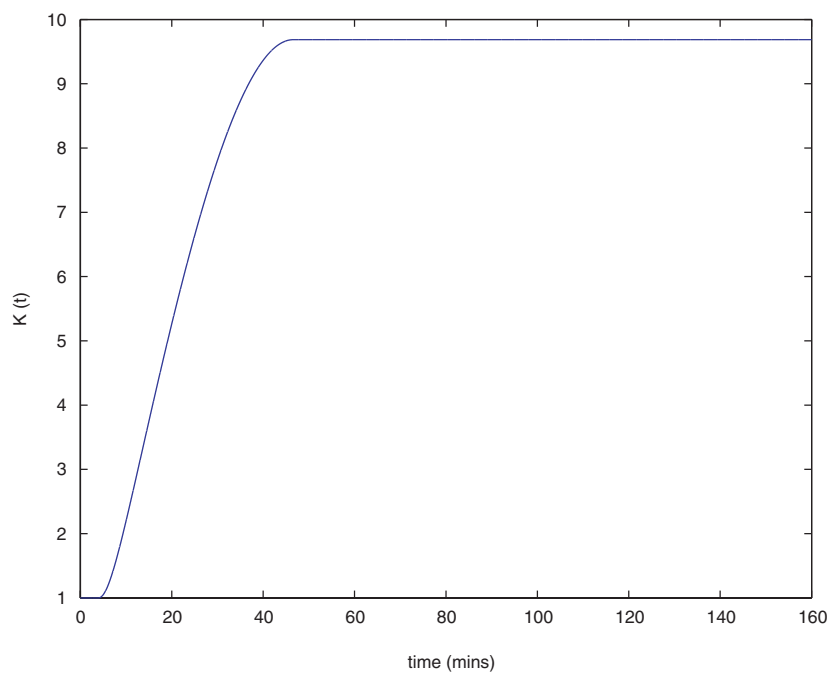

Figure 6. Adaptive gain.
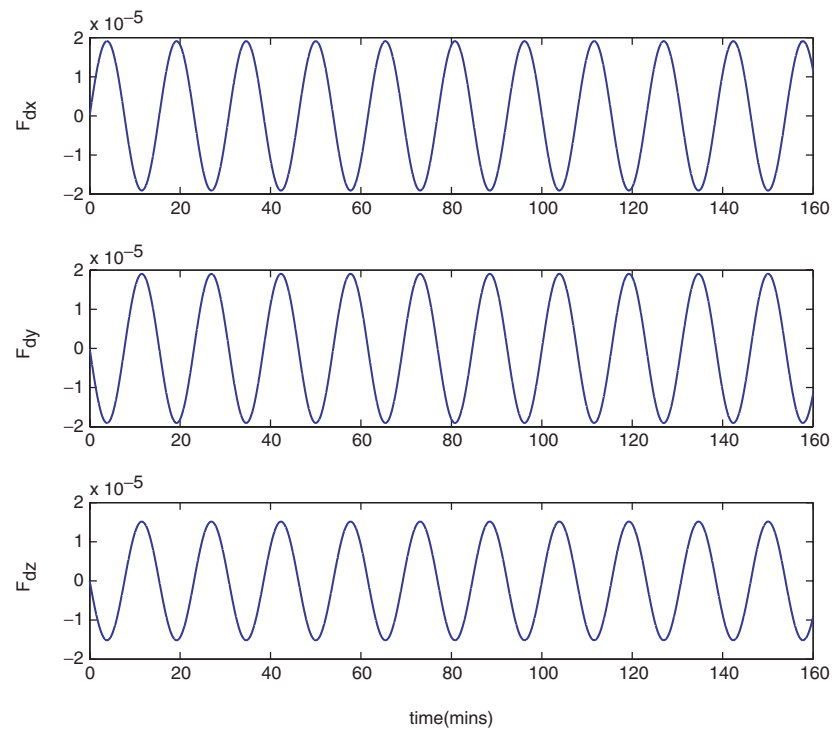

Figure 7. Disturbance.

mass variations of the satellites can be anticipated. Future work will examine the extension of this type of adaptive scheme to include attitude dynamics. Other on-going work is examining path planning for fuel economy.

\section{Acknowledgements}

This work is supported by EPSRC grant No. GR/ S25081/01(P) (constrained control methods for autonomous satellite formation flying systems) and was 
undertaken during a period when the first author was employed as a research fellow at the University of Southampton.

\section{References}

G.W. Hill, "Researches in lunar theory", American Journal of Mathematics, 1878, 1, 5-26.

C.L. Leonard, W.M. Hollister and E.V. Bergmann, "Orbital formation keeping with differential drag", AIAA Journal of Guidance, Dynamics and Control, 1999, 22, 108-113.

W. Lin and R. Pongvuthithum, "Adaptive output tracking of inherently nonlinear systems with nonlinear parameterization", IEEE Transactions on Automatic Control, 2003, 48, 1737-1349.

C.R. McInnes, "Autonomous proximity manoeuvering using artificial potential functions", ESA Journal, 1993, 17, 159-169.
M.S. de Queiroz, V. Kapila and Q. Yan, "Adaptive nonlinear control of multiple spacecraft formation flying", AIAA Journal of Guidance, Dynamics and Control, 2000, 23, 385-390.

C. Sabol, R. Burns and McLaughlin, "Satellite formation flying design and evolution", AIAA Journal of Spacecraft and Rockets, 2001, 38, 270-277.

S.M. Veres, S.B. Gabriel, D.Q. Mayne and E. Rogers, "Analysis of formation flying control of a pair of nanosatellites", AIAA Journal of Guidance, Dynamics and Control, 2002, 25, 971-974.

H. Wong, H. Pan, M.S. de Queiroz and V. Kapila, "Adaptive learning control for spacecraft formation flying", Proceedings of 40th IEEE Conference on Decision and Control, Orlando, FL, USA, 2001, pp. 1089-1094.

H.-H. Yeh, E. Nelson and A. Sparks, "Nonlinear tracking control for satellite formations", Proceedings of 39th IEEE Conference on Decision and Control, Sydney, Australia, 2000, pp. 328-333. 